\title{
The Thermodynamic Efficiency in Static and Dynamic Black Holes
}

\author{
Kh. Jafarzade ${ }^{a *}$, J. Sadeghi ${ }^{a \dagger}$ \\ ${ }^{a}$ Sciences Faculty, Department of Physics, University of Mazandaran, \\ P.O.Box 47415-416, Babolsar, Iran
}

October 31, 2017

\begin{abstract}
We note that, in extended phase space the cosmological constant is a thermodynamic variable. In this paper, this cosmological constant lead us to consider a black hole as a heat engine. So, here we take advantage from holographic heat engine and study two kind of different black holes. We first investigate a static black hole (Dyonic $\mathrm{BH}$ ) and consider the necessary condition to have high efficiency. Also we continue our investigation for dynamic black hole (rotating charged black hole) and study the effect of rotating parameter on the thermodynamic efficiency of holographic heat engine. We show that the rotating parameter has a more effective role than electric charge in thermodynamic efficiency.
\end{abstract}

Keywords: Static black hole; Dynamic black hole; Holography heat engines; Maximum efficiency.

\section{Introduction}

first time the thermodynamics of black holes was introduced by Hawking, Carter and Bardeen. They believed that black holes are thermodynamics and their temperature is related to event horizon [1]. It means that dynamic laws of black holes are similar to thermodynamic laws [2]. The similarity of four mechanic laws of black holes and thermodynamic laws formulated by Hawking, Carter, Bardeen and Chistodoulou [3-4]. In classical view of point, parameters of black hole as mass $M$, surface gravity $\kappa$ and area $A$ relate to the energy $U$, temperature $T$ and entropy $S$ of thermodynamic system which are given by [5-8],

$$
M=U \quad, \quad T=\frac{\kappa}{2 \pi} \quad, \quad S=\frac{A}{4},
$$

\footnotetext{
*Email: kh.Jafarzadeh@stu.umz.ac.ir
}

†Email: pouriya@ipm.ir 
and this subject has been extended to include the pressure $P$ and volume $V$ of black hole [9-20]. The cosmological constant of space time relates to pressure as $P=-\frac{\Lambda}{8 \pi}$ and thermodynamic volume of black holes is defined as: $V=\left(\frac{\partial M}{\partial P}\right)_{S, \phi_{i}, J_{k}}$ (here we are using geometric units $G_{N}=\hbar=c=k=1$ ). The black holes may have other parameters such as gauge charge $q_{i}$ and angular momentum $J_{j}$ with their conjugate, as potential $\phi_{i}$ and angular velocity $\Omega_{j}$ respectively.

Recently, the variation of cosmological constant $\Lambda$, in the first law of black holes thermodynamics has been attention by Ref.s. [21]. This lead us to consider the pressure as a thermodynamic variable, where the mass will be enthalpy [14,22],

$$
M=H \equiv U+P V .
$$

The first law of black holes thermodynamics in an extended phase space in four dimensions with an electric charge and rotation is,

$$
d M=T d S+V d P+\Phi d q+\Omega d J
$$

when $P$ is treated as constant (cosmological constant is not allowed to vary), the above relation reduces to the standard first law in the "non-extended" phase space. The thermodynamic volume of static black holes is equal to volume of horizon radius. For example in 4-dimensional Reissner- Nordstrom and Schwarzchild black holes we have following equation,

$$
V=\frac{4}{3} \pi r_{H}^{3}
$$

where the entropy is related to horizon radius and volume in static black holes. Thermodynamics of black holes not only determines standard thermodynamic variables such as temperature and entropy but also has extensive phase structure in analogy with known non gravitational thermodynamic systems and also it admit critical phenomena. Historically, the study of thermodynamic properties of $A d S$ black hole was started by impressive article of Hawking and Page [23], they have shown a phase transition in phase space of $A d S$ Schwarzchild black hole (non rotating uncharged). Then papers about phase transition and critical phenomena of systems with more complicated background increased [24,25]. The critical behavior of $R N-A d S$ black hole in non extended phase space $(\Lambda=$ cte and $\mathrm{P}=$ cte), at canonical ensemble (fixed charge) was studied in $[26,27]$ that was observed phase transition behavior is similar to Liquid-gas phase transition. Also, the critical behavior of this black hole in extended phase space is remarkably coincidence with Van der Waals fluid studied by [28]. In Ref. [29], authors considered a Dyonic black hole in $(3+1)$ dimensions (this black hole has a magnetic charge in additional to electric charge) and they have shown that putting black hole into ensemble of fixed electric charge and magnetic charge the obtained results is similar to [25-27] except the charge is redefined by $q_{E}^{2} \rightarrow q_{E}^{2}+q_{M}^{2}$. The quantum behavior of this black hole was studied in Ref.[30]. In quantum approach, the effect of thermal fluctuations is considered. Thermal fluctuations arise due to quantum fluctuations in the geometry of space-time and they appear in the black hole entropy as logarithmic term [31-34]. But its not our purpose in this paper. Here, we study thermodynamic black hole 
from the classical point of view. After studying of phase transition, it is interesting to define classical cycles for black holes like usual thermodynamic systems. It means that when small black hole translate to large black hole we need again the large black hole translate to small black hole, in other words the system must be back to primary state. On the other hand, the holographic heat engine has been studied for charge $A d S$ black hole [35]. This paper and above information give us motivation to study heat engine in dynamic black hole and compare with static black hole. In this paper first of all we are going to consider Static and Dynamic black holes and review some thermodynamic properties as thermodynamic cycles and heat engines generally. These thermodynamic cycles and heat engines help us to arrange black hole as Carnot cycle and obtain the corresponding efficiency for the static and dynamic black holes.

\section{Thermodynamic cycles and heat engines}

By using volume, pressure, temperature and entropy we can calculate heat energy and mechanical useful work. We start with equation of state (function of $P(V, T)$ ) and define an engine as a close path in $P-V$ plane which receives $Q_{H}$ and gives $Q_{C}$. From the first law of thermodynamics, total mechanical work is defined as, $W=Q_{H}-Q_{C}$. Therefore, the efficiency of heat engine is, $\eta=\frac{W}{Q_{H}}=1-\frac{Q_{C}}{Q_{H}}$.

Some of the classic cycles involve a pair of isotherms at temperature $T_{H}$ and $T_{C}\left(T_{H}>T_{C}\right)$ where there are isothermal expansion and compression while some heat is absorbed and some heat is exited respectively. By using different methods one can relate two systems with each other. The first method here is isochoric path like classical Stirling cycle and the second one is adiabatic path like classical Carnot cycle.

We know that a whole heat engine is fully reversible (since the total entropy flow zero). Therefore, we note here the maximum efficiency will be Carnot efficiency $\left(\eta=1-\frac{T_{C}}{T_{H}}\right)$. Any higher efficiency would violate the second Law. It is very important that how can reach to such efficiency in heat engine of black holes to preserve the second law. So, the form of path for the definition of cycle is important. As we know, in the static black holes the thermodynamic volume $V$ and entropy $S$ are not independent. It means that adiabats and isochores are the same, in that case Carnot and Stirling coincide to each other. So, the efficiency of cycle can be calculated easily.

So along the upper isotherm, we have the following heat flow,

$$
Q_{H}=T_{H} \Delta S_{1 \rightarrow 2}=T_{H}\left(\frac{3}{4 \pi}\right)^{\frac{2}{3}} \pi\left(V_{2}^{\frac{2}{3}}-V_{1}^{\frac{2}{3}}\right),
$$

and also along the lower isotherm the heat flow will be as,

$$
Q_{C}=T_{C} \Delta S_{3 \rightarrow 4}=T_{C}\left(\frac{3}{4 \pi}\right)^{\frac{2}{3}} \pi\left(V_{3}^{\frac{2}{3}}-V_{4}^{\frac{2}{3}}\right) .
$$

Since $V_{1}=V_{4}$ and $V_{2}=V_{3}$, the efficiency becomes,

$$
\eta=1-\frac{T_{C}}{T_{H}}
$$




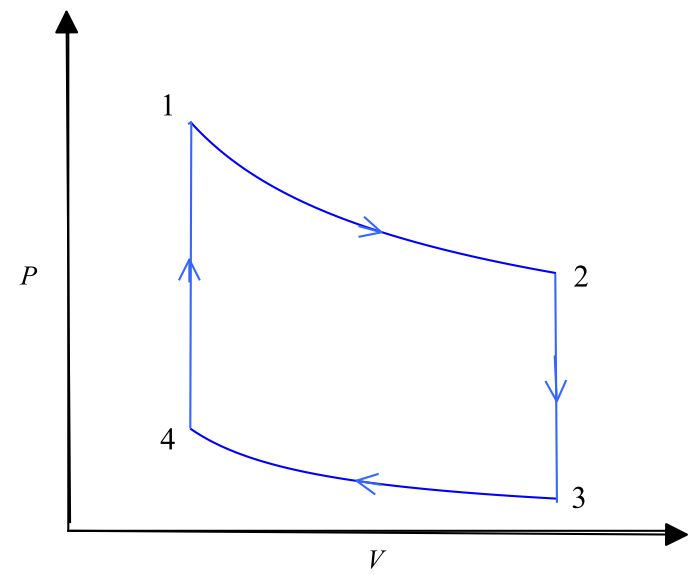

Figure 1: The Carnot engine.

So, we take advantage from above thermodynamic cycles and heat engines information and study the static and dynamic black hole.

\section{Thermodynamical properties of static black hole and heat engine}

Now we are going to consider the static black hole system (dyonic black hole). The solution of dyonic-dilaton $A d S$ black hole can be used for maximal gauge supergravity in 4-dimension [36]. In that case the corresponding action will be as,

$$
I=\frac{1}{16 \pi G_{4}} \int d^{4} x \sqrt{g}\left(-R+F^{2}-\frac{6}{b^{2}}\right) .
$$

The equation of motion is given by,

$$
\begin{aligned}
R_{\mu \nu}-\frac{1}{2} g_{\mu \nu} R-\frac{3}{b^{2}} g_{\mu \nu} & =2\left(F_{\mu \lambda} F_{\nu}^{\lambda}-\frac{1}{4} g_{\mu \nu} F_{\alpha \beta} F^{\alpha \beta}\right) ; \\
\nabla_{\mu} F^{\mu \nu} & =0 .
\end{aligned}
$$

One can write a static spherically symmetric solution to this action which is given by,

$$
d s^{2}=-f(r) d t^{2}+\frac{1}{f(r)} d r^{2}+r^{2} d \theta^{2}+r^{2} \sin ^{2} \theta d \phi^{2}
$$

where

$$
f(r)=\left(1+\frac{r^{2}}{b^{2}}-\frac{2 M}{r}+\frac{q_{E}^{2}+q_{M}^{2}}{r^{2}}\right) .
$$


The electromagnetic four-potential $A_{\mu}$ is,

$$
A=\left(-\frac{q_{E}}{r}+\frac{q_{E}}{r_{+}}\right) d t+\left(q_{M} \cos \theta\right) d \phi
$$

where $q_{E}, q_{M}$ and $M$ are electric, magnetic charge and mass of black hole respectively. The horizon of black hole is given by,

$$
f\left(r_{+}\right)=\left(1+\frac{r_{+}^{2}}{b^{2}}-\frac{2 M}{r_{+}}+\frac{q_{E}^{2}+q_{M}^{2}}{r_{+}^{2}}\right)=0,
$$

and electric potential $\Phi_{E}$ is defined by following equation,

$$
\Phi_{E}=\frac{q_{E}}{r_{+}} .
$$

The Hawking temperature of this black hole is following,

$$
T=\frac{1}{\beta}=\frac{1}{4 \pi r_{+}}\left[1+\frac{3 r_{+}^{2}}{b^{2}}-\Phi_{E}^{2}-\frac{q_{M}^{2}}{r_{+}^{2}}\right] .
$$

The pressure is given by $[28,37]$,

$$
P=-\frac{\Lambda}{8 \pi}=\frac{3}{8 \pi} \frac{1}{b^{2}}
$$

and the thermodynamical volume is,

$$
V=\frac{4 \pi}{3} r_{+}^{3}
$$

We can write the temperature in equation (15) in terms of $S$ and $P$ as follows,

$$
T=\frac{1}{4 \sqrt{\pi}} \frac{1}{\sqrt{S}}\left(1+8 P S-\Phi_{E}^{2}-\frac{\pi q_{M}^{2}}{S}\right) .
$$

The equation of state is given by,

$$
P=\frac{T}{v}-\frac{1-\Phi_{E}^{2}}{2 \pi v^{2}}+\frac{2 q_{M}^{2}}{\pi v^{4}}
$$

where $v=2 r_{+}$can be identified with the specific volume of the system [29]. This equation describes different phases of a dyonic black hole in a fixed electric potential and magnetic charge ensemble which is similar to extended liquid-gas phase diagram. In thermodynamic, the heat capacity is an important measurable physical quantity. It determine the amount of requisite heat to change the temperature of an object by a given amount. There are two different heat capacities for a system, heat capacity at constant pressure and heat capacity at constant volume. Heat capacity can be calculated by the standard thermodynamic relations, which is given by,

$$
C_{V}=\left.T \frac{\partial S}{\partial T}\right|_{V} \quad, \quad C_{P}=\left.T \frac{\partial S}{\partial T}\right|_{P} .
$$



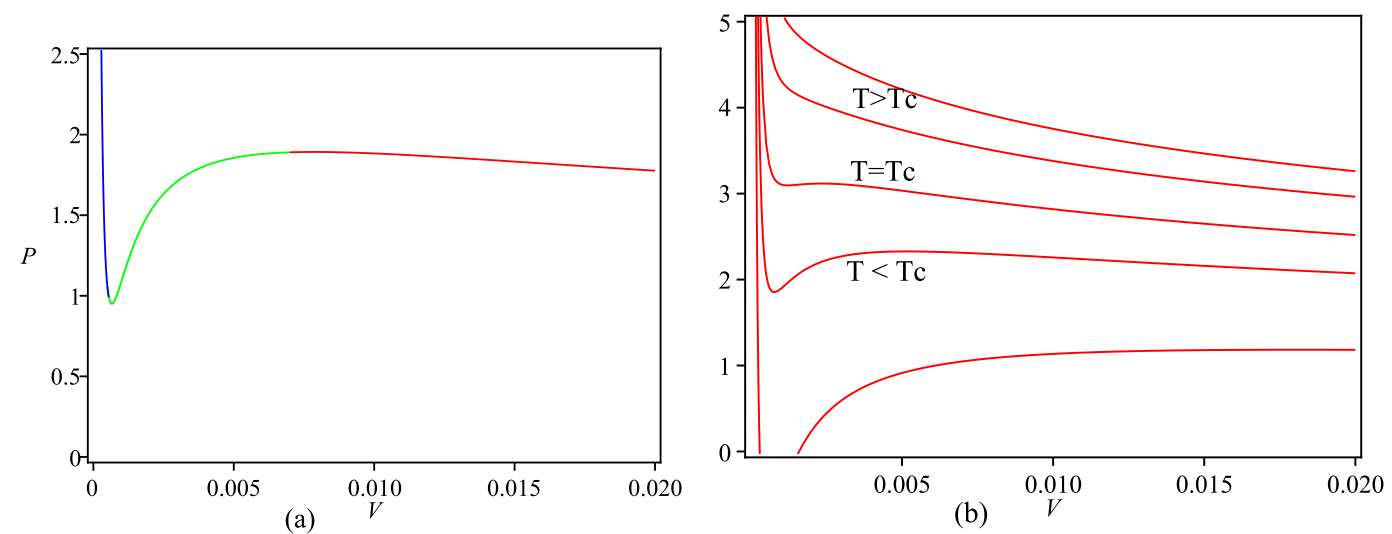

Figure 2: (a) P-V diagram for all other parameters held constant $\left(q_{M}=0.028, \Phi_{E}=0.35\right.$, $T=1.05)$. (b) P-V diagram for fixed $\left(q_{M}=0.028, \Phi_{E}=0.35\right)$ and varying $\mathrm{T}$.

The entropy is expressed as,

$$
S=\frac{A_{H}}{4}=\pi r_{+}^{2}=\pi\left(\frac{3 V}{4 \pi}\right)^{\frac{2}{3}} .
$$

By using Eq. (20) and Eq. (21) we have $C_{V}=0$ and $C_{P}$ which is given by,

$$
C_{P}=2 S \frac{\left(8 P S^{2}+S\left(1-\Phi_{E}^{2}\right)-\pi q_{M}^{2}\right)}{\left(8 P S^{2}+S\left(1-\Phi_{E}^{2}\right)+3 \pi q_{M}^{2}\right)} .
$$

One can write $P$ from equation (19) as a function of thermodynamical volume, so we have,

$$
P=\frac{T}{V^{\frac{1}{3}}}\left(\frac{\pi}{6}\right)^{\frac{1}{3}}-\frac{1-\Phi_{E}^{2}}{2 \pi V^{\frac{2}{3}}}\left(\frac{\pi}{6}\right)^{\frac{2}{3}}+\frac{2 q_{M}^{2}}{\pi V^{\frac{4}{3}}}\left(\frac{\pi}{6}\right)^{\frac{4}{3}} .
$$

In $P-V$ diagram we fixed $\left(T, \Phi_{E}, q_{M}\right)$, so in that case we draw $P$ with respect $V$ as two figures $2 a$ and $2 b$. In figure (2a), there are three different solutions for static black hole horizon. These are identified by branch-1 (blue), branch-2 (green) and branch-3 (red). The small black hole (SBH) just exist for the large $P$, so we have branch-1. For the low $P$, we only have the large black hole (LBH) as branch-3. On the other hand, the branch-2 indicates thermodynamical unstable phase. Now we back to figure (2b) and see that the branch-2 is disappeared by the condition $\left(T>T_{c}\right)$ and phase transition is happened for $T<T_{C}$. In phase transition, $\mathrm{SBH}$ reduces to $\mathrm{LBH}$. If $\mathrm{LBH}$ transfers to $\mathrm{SBH}$, we can define classical cycle for such black hole. The SBH absorbs heat $Q_{H}$ along isothermal expansion and exists at high pressure. Actually, an explicit expression $C_{P}$ lead us to have a new engine which include two isobars and two isochores/adiabatas as figure(3). The work done in this cycle is, 


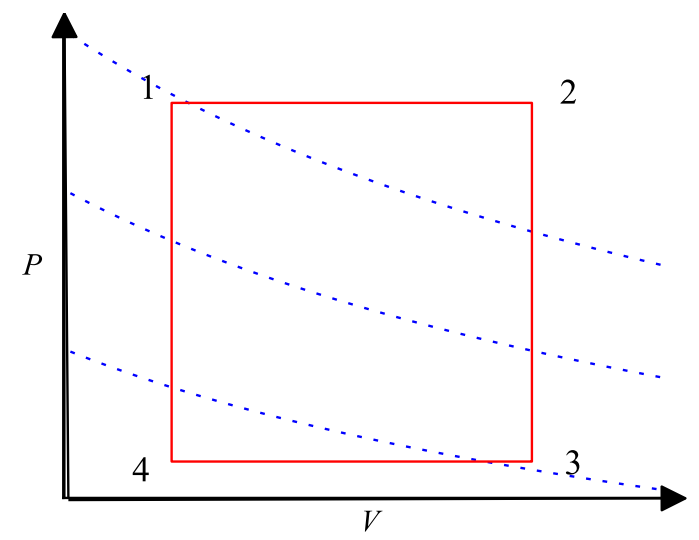

Figure 3: Our other engine.

$$
\begin{aligned}
& W=\oint P d V \\
& W_{\text {total }}=W_{1 \rightarrow 2}+W_{3 \rightarrow 4}=P_{1}\left(V_{2}-V_{1}\right)+P_{4}\left(V_{4}-V_{3}\right) \\
& W_{\text {total }}=\frac{4}{3 \sqrt{\pi}}\left(P_{1}-P_{4}\right)\left(S_{2}^{\frac{3}{2}}-S_{1}^{\frac{3}{2}}\right) .
\end{aligned}
$$

The upper isobar will give us following heat $Q_{H}$,

$$
Q_{H}=\int_{T_{1}}^{T_{2}} C_{P}\left(P_{1}, T\right) d T .
$$

The corresponding entropy and $C_{P}$ in high pressure and temperature are given by,

$$
\begin{aligned}
S & =\frac{\pi T^{2}}{4 P^{2}}-\frac{\left(1-\Phi_{E}^{2}\right)}{4 P}-\frac{\left(1-\Phi_{E}^{2}\right)^{2}}{16 \pi T^{2}}+\ldots \\
C_{P} & =\frac{\pi T^{2}}{2 P^{2}}-\frac{\left(1-\Phi_{E}^{2}\right)}{2 P}-\frac{\left(1-\Phi_{E}^{2}\right)^{2}}{8 \pi T^{2}}+\ldots
\end{aligned}
$$

and

$$
Q_{H}=\frac{4 P_{1}}{3 \sqrt{\pi}}\left(S_{2}^{\frac{3}{2}}-S_{1}^{\frac{3}{2}}\right)-\frac{\left(1-\Phi_{E}^{2}\right)}{\sqrt{\pi}}\left(S_{2}^{\frac{1}{2}}-S_{1}^{\frac{1}{2}}\right)+\frac{\left(1-\Phi_{E}^{2}\right)^{2}}{16 \sqrt{\pi} P_{1}}\left(S_{2}^{-\frac{1}{2}}-S_{1}^{-\frac{1}{2}}\right) .
$$

The above equation help us to arrange the efficiency for the static black hole which is obtained by,

$$
\eta=\left(1-\frac{T_{C}}{T_{H}}\right)\left\{1-\frac{3\left(\Phi_{E}^{2}-1\right)\left(S_{2}^{\frac{1}{2}}-S_{1}^{\frac{1}{2}}\right)}{4 P_{1}\left(S_{2}^{\frac{3}{2}}-S_{1}^{\frac{3}{2}}\right)}+O\left(\frac{1}{P_{1}^{2}}\right)\right\} .
$$

As we mentioned before the maximum efficiency always coming from Carrnot cycle, here also we had maximum efficiency. So, one can employed Carrnot efficiency at high pressure limit and for values of $\Phi_{E}$ close to 1 . 


\section{Thermodynamical properties of dynamic black hole and heat engine}

Now we investigate dynamic black hole system. One example of such a system is $A d S$ rotating charged black hole solution which the corresponding metric is given by [38],

$$
d s^{2}=-\frac{\Delta}{\rho^{2}}\left[d t-\frac{a \sin ^{2} \theta}{\Xi} d \varphi\right]^{2}+\frac{\rho^{2}}{\Delta} d r^{2}+\frac{\rho^{2}}{S} d \theta^{2}+S \frac{\sin ^{2} \theta}{\rho^{2}}\left[a d t-\frac{r^{2}+a^{2}}{\Xi} d \varphi\right]^{2},
$$

where in $d=4$ we have following expressions,

$$
\rho^{2}=r^{2}+a^{2} \csc ^{2} \theta, \quad \Xi=1-\frac{a^{2}}{\ell^{2}}, \Delta=\left(r^{2}+a^{2}\right)\left(1+\frac{r^{2}}{\ell^{2}}\right)-2 m r+q^{2}, \quad s=1-\frac{a^{2}}{\ell^{2}} \csc ^{2} \theta .
$$

The entropy, temperature, electric potential and angular velocity are given by,

$$
S=\pi \frac{\left(r_{+}^{2}+a^{2}\right)}{\Xi} \quad, \quad T=\frac{r_{+}\left(1+\frac{a^{2}}{\ell^{2}}+\frac{3 r_{+}^{2}}{\ell^{2}}-\frac{a^{2}+q^{2}}{r_{+}^{2}}\right)}{4 \pi\left(r_{+}^{2}+a^{2}\right)}, \Phi=\frac{q r_{+}}{r_{+}^{2}+a^{2}} \quad, \quad \Omega_{H}=\frac{a \Xi}{r_{+}^{2}+a^{2}},
$$

one can rewrite the temperature as follows,

$$
T=\frac{r_{+}}{2 S}-\frac{1}{4 \pi r_{+}}+2 P r_{+}-\frac{q^{2}}{4 \Xi S r_{+}}
$$

The mass of black hole $M$, the charge $Q$ and the angular momentum $J$ are related to parameters $m, q$ and $a$ as follows,

$$
M=\frac{m}{\Xi^{2}} \quad, \quad Q=\frac{q}{\Xi} \quad, \quad J=\frac{a m}{\Xi^{2}} .
$$

The pressure is identified by, $P=-\frac{\Lambda}{8 \pi}=\frac{3}{8 \pi} \frac{1}{\ell^{2}}$ and the thermodynamic volume is $[15,17]$,

$$
V=\frac{2 \pi}{3} \frac{\left(r_{+}^{2}+a^{2}\right)\left(2 r_{+}^{2} \ell^{2}+a^{2} \ell^{2}-r_{+}^{2} a^{2}\right)+\ell^{2} q^{2} a^{2}}{\ell^{2} \Xi^{2} r_{+}}
$$

The equation of state is written by following expression,

$$
P=\frac{T}{v}-\frac{1}{2 \pi v^{2}}+\frac{2 Q^{2}}{\pi v^{4}}+\frac{48 J^{2}}{\pi v^{6}}-\frac{96 Q^{2}\left(24 Q^{2}+5 v^{2}+6 \pi T v^{3}\right) J^{2}}{\pi v^{6}\left(8 Q^{2}+v^{2}+\pi T v^{3}\right)^{2}}
$$

where

$$
v=2\left(\frac{3 V}{4 \pi}\right)^{\frac{1}{3}}=2 r_{+}+\frac{12\left(3 r_{+}^{2}+8 \pi r_{+}^{4} P+Q^{2}\right) J^{2}}{r_{+}\left(3 r_{+}^{2}+8 \pi r_{+}^{4} P+3 Q^{2}\right)^{2}}
$$

Now we are going to investigate the thermodynamical properties for dynamic black hole (rotating black hole) with electric charge and without charge. First of all we consider zero charge as $Q=0$. In that case the equation of state is expressed as follows,

$$
P=\frac{T}{v}-\frac{1}{2 \pi v^{2}}+\frac{48 J^{2}}{\pi v^{6}},
$$




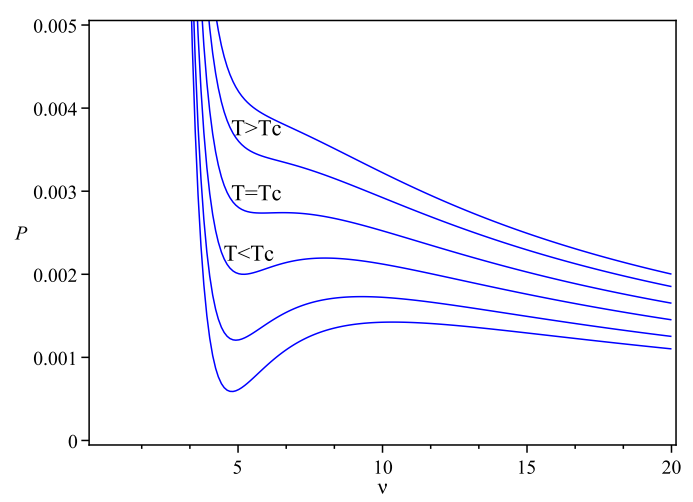

Figure 4: $P-v$ diagram for the rotating black hole for fixed $\mathrm{J}=1$ and varying $\mathrm{T}$.

where

$$
v=2\left(\frac{3 V}{4 \pi}\right)^{\frac{1}{3}}=2 r_{+}+\frac{12 J^{2}}{r_{+}\left(3 r_{+}^{2}+8 \pi r_{+}^{4} P\right)} .
$$

In figure (4), we draw pressure with respect to specific volume. In this figure, we have some phase transition and one can easily define the holography heat engine for dynamic black hole (rotating black hole). So, this property lead us to investigate corresponding black hole as Carnot cycle and obtain the efficiency parameter. For this reason, we have to prepare some thermodynamic quantities as heat capacity, useful work and heat $Q_{H}$ to arrange corresponding efficiency.

First of all we are going to calculate the heat capacity. The heat capacity at constant pressure is given by,

$$
C_{P}=\frac{2\left(\Xi S-a^{2}\right)}{\Xi}\left(\frac{\frac{1}{2 S}-\frac{1}{4\left(\Xi S-a^{2}\right)}+2 P}{\frac{1}{2 S}+\frac{1}{4\left(\Xi S-a^{2}\right)}+2 P-\frac{\left(\Xi S-a^{2}\right)}{S \Xi^{2}}}\right) .
$$

By assuming $\frac{\pi a^{2}}{S \Xi} \ll 1$, we can rewrite thermodynamic volume as follows,

$$
V=\frac{2 S^{\frac{3}{2}}}{3 \sqrt{\pi \Xi}}\left((1+\Xi)+\frac{\pi a^{2}}{2 S \Xi}(1-\Xi)\right)
$$

As we see, the volume is a function of $S, a$ and $\ell$. Then one can say, these parameters will be constant at constant volume. On the other hand, the temperature is dependent on $S, a$ and $\ell$. So, $\frac{\partial T}{\partial S}$ is zero at constant volume $\left(C_{V}=0\right)$. Therefore we define a cycle as figure $(5)$. The work done along the isobars is,

$$
W=\left(\frac{2(1+\Xi)}{3 \sqrt{\pi \Xi}}\left(S_{2}^{\frac{3}{2}}-S_{1}^{\frac{3}{2}}\right)+\frac{a^{2}}{3} \sqrt{\frac{\pi}{\Xi^{3}}}(1-\Xi)\left(S_{2}^{\frac{1}{2}}-S_{1}^{\frac{1}{2}}\right)\right)\left(P_{1}-P_{4}\right) .
$$




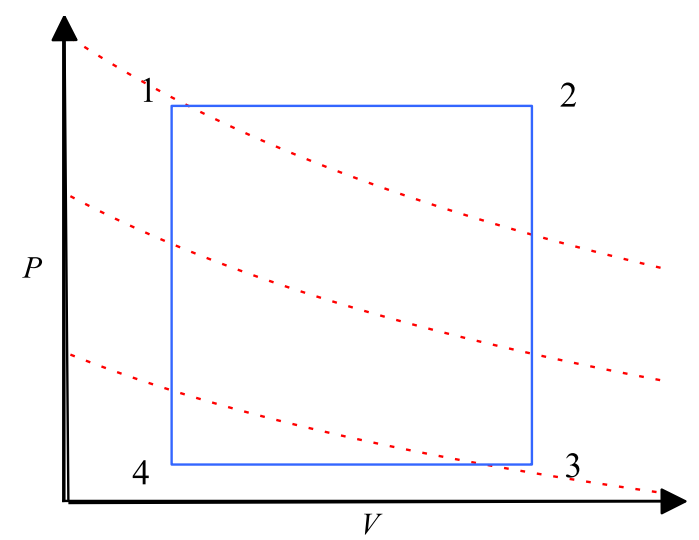

Figure 5: Our other engine.

We take the high temperature and pressure limit to have explicit expressions for the efficiency. Therefor, by expanding entropy and heat capacity at constant pressure and large $T$, we can obtain the following expressions,

$$
\begin{gathered}
S=\frac{\pi T^{2}}{4 \Xi P^{2}}-\frac{(2 \Xi-1)}{4 \Xi P}-\frac{(2 \Xi-1)^{2}}{16 \pi \Xi T^{2}}+\ldots \\
C_{P}=\frac{\pi T^{2}}{2 \Xi P^{2}}-\frac{(2 \Xi-1)}{2 \Xi P}-\frac{(2 \Xi-1)^{2}}{8 \pi \Xi T^{2}}+\ldots,
\end{gathered}
$$

Consequently, the heat $Q_{H}$ is as follows,

$$
\left.Q_{H}=\frac{4 P_{1}}{3} \sqrt{\frac{\Xi}{\pi}}\left(S_{2}^{\frac{3}{2}}-S_{1}^{\frac{3}{2}}\right)-\frac{(2 \Xi-1)}{\sqrt{\pi \Xi}}\left(S_{2}^{\frac{1}{2}}-S_{1}^{\frac{1}{2}}\right)+\frac{(2 \Xi-1)^{2}}{16 P_{1} \sqrt{\pi \Xi^{3}}}\left(S_{2}^{-\frac{1}{2}}-S_{1}^{-\frac{1}{2}}\right)\right) .
$$

Thus, the efficiency of this cycle is,

$$
\eta=\left(1-\frac{T_{C}}{T_{H}}\right)\left(\frac{1+\Xi}{2 \Xi}\right)(1-\beta)
$$

where

$$
\beta=\frac{\pi a^{2}(\Xi-1)}{2 \Xi(\Xi+1)} \frac{\left(S_{2}^{\frac{1}{2}}-S_{1}^{\frac{1}{2}}\right)}{\left(S_{2}^{\frac{3}{2}}-S_{1}^{\frac{3}{2}}\right)}+\frac{3(1-2 \Xi)}{4 \Xi P_{1}} \frac{\left(S_{2}^{\frac{1}{2}}-S_{1}^{\frac{1}{2}}\right)}{\left(S_{2}^{\frac{3}{2}}-S_{1}^{\frac{3}{2}}\right)}+\ldots
$$

From Eqs. (44) and (45), it is obvious that the defined cycle has the Carrnot efficiency for very small values of rotating parameter $(a \ll 1)$. Figure $(6)$ show the ratio $\frac{\eta}{\eta_{C}}$ with respect to $a$ for three values of pressure $\left(P_{1}=1, P_{1}=2\right.$ and $\left.P_{1}=5\right)$. We noted that, the corresponding black hole has not physical solution for $P_{1}<1$. And at $P_{1}=1$, the obtained efficiency is larger than the Carrnot efficiency in range of $0<a<0.48$ which is inconsistent with the second law of thermodynamic. But $a>0.48$ satisfy the second law. At $P_{1}=2$ and $P_{1}=5$, the obtained efficiency is consistent with the second law for $a>0.34$ and $a>0.21$ 


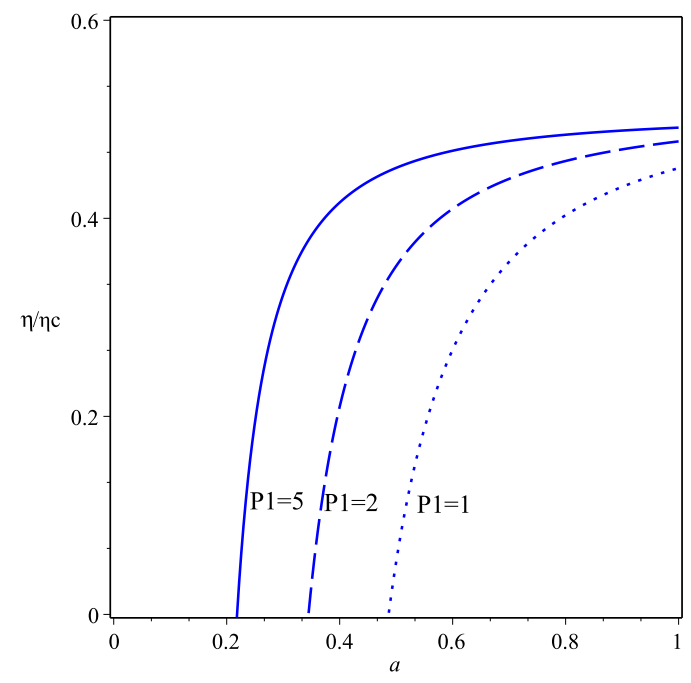

Figure 6: The ratio $\frac{\eta}{\eta_{C}}$ with respect to $a$ for fixed $\left(S_{2}=20\right.$ and $\left.S_{1}=10\right)$ and varying $P_{1}$.

respectively. It show that, the corresponding engine has a physical efficiency for any value of rotating parameter $a$ at large pressure limit. As we see for $a>1$, the efficiency reaches a specific value $\left(\eta_{\max } \simeq \frac{1}{2} \eta_{C}\right)$ which this consequence is independent of pressure value.

Now we are going to investigate the heat engine of corresponding solution in presence of electric charge as $(Q \neq 0)$. By using equation (35), we can investigate phase structure of rotating charged black hole. We draw pressure with respect to specific volume in figure (7). As we see, the corresponding black hole solution has a Van der Walss-like behavior. Therefore, we can define the holography heat engine for this solution. First of all we calculate the heat capacity at constant pressure, which is given by,

$$
C_{P}=\frac{2\left(\Xi S-a^{2}\right)}{\Xi}\left(\frac{\frac{1}{2 S}-\frac{1}{4\left(\Xi S-a^{2}\right)}+2 P-\frac{\pi q^{2}}{4 \Xi S\left(\Xi S-a^{2}\right)}}{\frac{1}{2 S}+\frac{1}{4\left(\Xi S-a^{2}\right)}+2 P+\frac{\pi q^{2}}{4 \Xi S\left(\Xi S-a^{2}\right)}+\frac{\pi q^{2}}{2 \Xi^{2} S^{2}}-\frac{\left(\Xi S-a^{2}\right)}{S \Xi^{2}}}\right)
$$

Here, also we rewrite thermodynamic volume by assuming $\frac{\pi a^{2}}{S \Xi} \ll 1$,

$$
V=\frac{2 S^{\frac{3}{2}}}{3 \sqrt{\pi \Xi}}\left((1+\Xi)+\frac{\pi a^{2}}{2 S \Xi}(1-\Xi)+\frac{\pi^{\frac{3}{2}} q^{2} a^{2}}{\Xi^{\frac{5}{2}} S^{\frac{1}{2}}}\right)
$$

Here also we will have $C_{V}=0$ similar to pervious case $(Q=0)$. In that case, we can define a cycle as figure (8) and obtain the work done along the isobars,

$W=\left(\frac{2(1+\Xi)}{3 \sqrt{\pi \Xi}}\left(S_{2}^{\frac{3}{2}}-S_{1}^{\frac{3}{2}}\right)+\frac{a^{2}}{3} \sqrt{\frac{\pi}{\Xi^{3}}}(1-\Xi)\left(S_{2}^{\frac{1}{2}}-S_{1}^{\frac{1}{2}}\right)+\frac{2 \pi^{\frac{3}{2}} q^{2} a^{2}}{3 \Xi^{\frac{5}{2}} S^{\frac{1}{2}}}\left(S_{2}^{-\frac{1}{2}}-S_{1}^{-\frac{1}{2}}\right)\right)\left(P_{1}-P_{4}\right)$. 


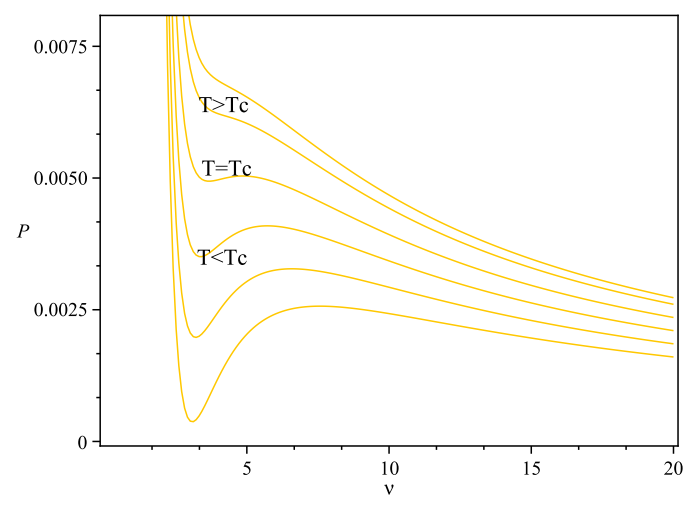

Figure 7: $P-v$ diagram for the rotating charged black hole for fixed $\mathrm{q}=0.5, \mathrm{~J}=0.4$ and varying $\mathrm{T}$.

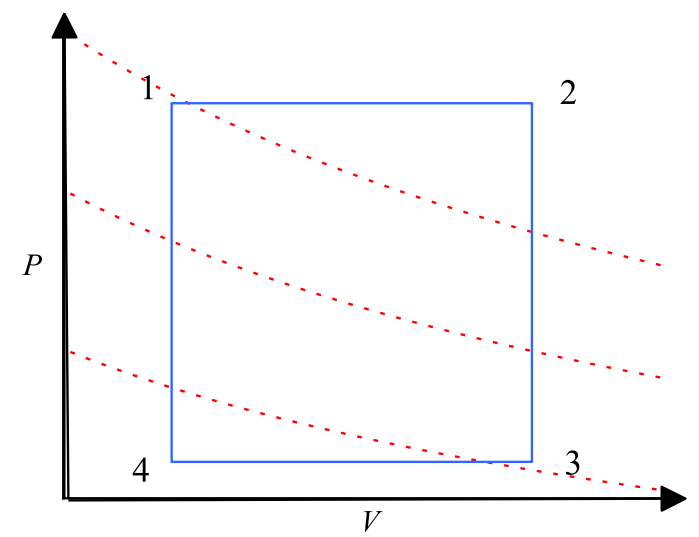

Figure 8: Our other engine.

As before, we obtain $S$ and $C_{P}$ by expanding around large pressure and temperature,

$$
\begin{gathered}
S=\frac{\pi T^{2}}{4 \Xi P^{2}}-\frac{(2 \Xi-1)}{4 \Xi P}-\frac{(2 \Xi-1)^{2}}{16 \pi \Xi T^{2}}+\ldots \\
C_{P}=\frac{\pi T^{2}}{2 \Xi P^{2}}-\frac{(2 \Xi-1)}{2 \Xi P}-\frac{(2 \Xi-1)^{2}}{8 \pi \Xi T^{2}}+\ldots
\end{gathered}
$$

Consequently, the heat $Q_{H}$ is as follows,

$$
\left.Q_{H}=\frac{4 P_{1}}{3} \sqrt{\frac{\Xi}{\pi}}\left(S_{2}^{\frac{3}{2}}-S_{1}^{\frac{3}{2}}\right)-\frac{(2 \Xi-1)}{\sqrt{\pi \Xi}}\left(S_{2}^{\frac{1}{2}}-S_{1}^{\frac{1}{2}}\right)+\frac{(2 \Xi-1)^{2}}{16 P_{1} \sqrt{\pi \Xi^{3}}}\left(S_{2}^{-\frac{1}{2}}-S_{1}^{-\frac{1}{2}}\right)\right) .
$$

Therefore, the efficiency of this cycle is,

$$
\eta=\left(1-\frac{T_{C}}{T_{H}}\right)\left(\frac{1+\Xi}{2 \Xi}\right)(1-\beta),
$$



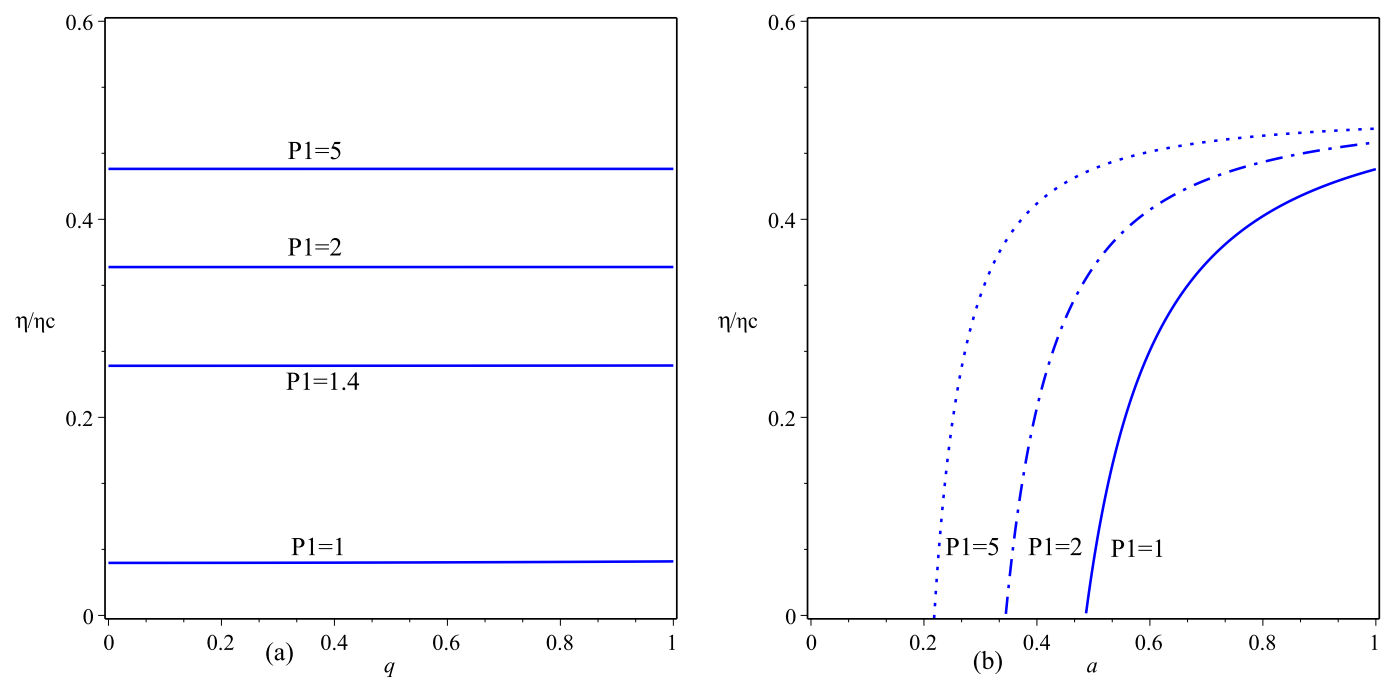

Figure 9: (a) The ratio $\frac{\eta}{\eta_{C}}$ with respect to $q$ for fixed ( $a=0.5, S_{2}=20$ and $\left.S_{1}=10\right)$ and varying $P_{1}$. (b) The ratio $\frac{\eta}{\eta_{C}}$ with respect to $a$ for fixed $\left(q=0.5, S_{2}=20\right.$ and $\left.S_{1}=10\right)$ and varying $P_{1}$.

where

$$
\beta=\frac{\pi a^{2}(\Xi-1)}{2 \Xi(\Xi+1)} \frac{\left(S_{2}^{\frac{1}{2}}-S_{1}^{\frac{1}{2}}\right)}{\left(S_{2}^{\frac{3}{2}}-S_{1}^{\frac{3}{2}}\right)}+\frac{3(1-2 \Xi)}{4 \Xi P_{1}} \frac{\left(S_{2}^{\frac{1}{2}}-S_{1}^{\frac{1}{2}}\right)}{\left(S_{2}^{\frac{3}{2}}-S_{1}^{\frac{3}{2}}\right)}-\frac{\pi^{2} q^{2} a^{2}}{\Xi^{2}(\Xi+1)} \frac{\left(S_{2}^{-\frac{1}{2}}-S_{1}^{-\frac{1}{2}}\right)}{\left(S_{2}^{\frac{3}{2}}-S_{1}^{\frac{3}{2}}\right)}+\ldots
$$

The obtained efficiency is same to equation (44) and the only difference is the term of electric charge. In figure (9a), we considered the rotating parameter as a constant and draw the ratio $\frac{\eta}{\eta_{C}}$ with respect to $q$. As we see, the efficiency increases by increasing pressure and it doesn't change by varying the charge. Then we include that the charge of black hole has any role in efficiency of this black hole solution. We also draw the ratio $\frac{\eta}{\eta_{C}}$ with respect to $a$ for fixed $q$ in figure (9b). The figure is exactly identical to figure (6). By comparing figures (9a) and (9b), we noted that the rotating parameter $a$ has a more effective role than $q$ in the corresponding efficiency.

\section{Conclusion}

In this paper we have studied thermodynamic cycle and heat engine for different black holes. We considered the holographic heat engine for a static black hole (Dyonic BH) in constant electric potential and magnetic charge ensemble. We noticed that at high pressure and temperature limit, the corresponding heat engine has a maximum efficiency. Then we continued our investigation and considered a dynamic black hole (rotating charged black hole). For first case (the solution without electric charge), We saw that, the physical solution existed for only $P_{1}>1$. And at large pressure limit the corresponding engine had a physical efficiency 
for any value of rotating parameter. Also we noted that, the corresponding efficiency will have maximum value $\left(\eta_{\max } \simeq \frac{1}{2} \eta_{C}\right)$ for $a>1$. Then we considered a rotating black hole along with electric charge and defined a cycle for the corresponding solution. We saw the obtained result of rotating charged black hole is identical to rotating black hole. In other words the efficiency is independent of charge value and only the rotating parameter $a$ has a key role in corresponding efficiency.

\section{References}

[1] S. W. Hawking, Nature (London) 248 (1974) 30.

[2] A. B. Nielsen, Gen. Rel. Grav. 41(2009)1539.

[3] D. Christodoulou, Phys. rev. Lett. 25 (1971) 1596.

[4] J. D. Bekenstein, Phys. rev. D 7 (1973) 949.

[5] J. D. Bekenstein, Phys. Rev. D 7 (1973) 2333.

[6] S. Hawking, Commun. Math. Phys. 43 (1975) 199.

[7] J. D. Bekenstein, Phys. Rev. D 9 (1974) 3292.

[8] S. Hawking, Phys. Rev. D 13 (1976) 191.

[9] M. M. Caldarelli, G. Cognola, and D. Klemm, Class. Quant. Grav. 17 (2000) 399.

[10] S. Wang, S. Q. Wu, F. Xie and L. Dan, Chin. Phys. Lett. 23 (2006) 1096.

[11] Y. Sekiwa, Phys. Rev. D 73 (2006) 084009.

[12] E. A. Larranaga Rubio, Stringy Generalization of the First Law of Thermodynamics for Rotating BTZ Black Hole with a Cosmological Constant as State Parameter, arXiv:0711.0012 [gr-qc]].

[13] D. Kastor, S. Ray, and J. Traschen, Class. Quant. Grav. 26 (2009) 195011.

[14] B. P. Dolan, Class. Quant. Grav. 28 (2011) 125020.

[15] M. Cvetic, G. Gibbons, D. Kubiznak, and C. Pope, Phys. Rev. D 84 (2011) 024037.

[16] B. P. Dolan, Phys. Rev. D 84 (2011) 127503.

[17] B. P. Dolan, Class. Quant. Grav. 28 (2011) 235017.

[18] M. Henneaux and C. Teitelboim, Phys. Lett. B 143 (1984) 415.

[19] C. Teitelboim, Phys. Lett. B 158 (1985) 293. 
[20] M. Henneaux and C. Teitelboim, Phys. Lett. B 222 (1989) 195.

[21] H. Lu, Y. Pang, C. N. Pope and J. F. Vazquez-Poritz, Phys. Rev. D 86 (2012) 044011.

[22] J. Sadeghi, K. Jafarzade and B. Pourhassan, Thermodynamical Quantities of HoravaLifshitz Black Hole, Int. J. Theor. Phys. 51 (2012) 3891.

[23] S. Hawking and D. N. Page, Commun. Math. Phys. 87 (1983) 577.

[24] M. Cvetic and S. S. Gubser, JHEP 9904 (1999) 024.

[25] M. Cvetic and S. Gubser, JHEP 9907 (1999) 010.

[26] A. Chamblin, R. Emparan, C. Johnson and R. Myers, Phys. Rev. D 60 (1999) 064018.

[27] A. Chamblin, R. Emparan, C. Johnson and R. Myers, Phys. Rev. D 60 (1999) 104026.

[28] D. Kubiznak and R. B. Mann, JHEP 1207(2012) 033.

[29] S. Dutta, A. Jain and R. Soni, JHEP 12 (2013) 060.

[30] J. Sadeghi, B. Pourhassan and M. Rostami, P-V criticality of logarithm-corrected dyonic charged AdS black holes, phys. Rev. D 94 (2016) 064006.

[31] B. Pourhassan and M.Faizal, Thermodynamics of a Sufficient Small Singly Spinning Kerr-AdS Black Hole, Nucl. Phys. B 913 (2016) 834.

[32] B. Pourhassan, M. Faizal and U. Debnath, Effects of Thermal Fluctuations on the Thermodynamics of Modified Hayward Black Hole, Eur. Phys. J. C 76 (2016) 145.

[33] B. Pourhassan and M. Faizal, Thermal Fluctuations in a Charged AdS Black Hole, EPL 111 (2015) 40006.

[34] J. Sadeghi, B. Pourhassan and F. Rahimi, Logarithmic corrections to charged hairy black hole in $(2+1)$ dimensions, Canadian Journal of Physics, 92 (2014) 1638.

[35] C. V. Johnson, Class. Quant. Grav. 31 (2014) 205002.

[36] H. Lu, Y. Pang and C. N. Pope, JHEP 1311 (2013) 033.

[37] S. Upadhyay, B. Pourhassan and H. Farahani, P-V criticality of first-order entropy corrected AdS black holes in massive gravity, Phys. Rev. D 95 (2017) 106014.

[38] S. Gunasekaran, D. Kubiznak and R. B. Mann, JHEP 11 (2012) 110. 